究. 时珍国医国药，24(11)，2702-2704.

5. Navarro F, Sarabiam et al (2011). A 40-month multicentre, randomised placebo-controlled study to assess the efficacy and carry over effect of repeated intra-articular injections of hyaluronic acid in knee osteoarthritis: the AMELIA project. Ann Rheum Dis, 70, 1957- 1962.

6. Phạm Xuân Phong và cộng sự (2018). Đánh giá hiệu quả điều trị thoái hóa khớp gối của thuốc Hoàn chỉ thống. Tạp chí Y học Việt Nam, 471(1),
112-117.

7. Đố Thị Tuyến và cộng sự (2020). Đặc điểm lâm sàng và Xquang các bểnh nhân thoái hóa khớp gối điều trị tại Khoa Châm cứu dưỡng sinh/Viện Y học cổ truyền Quân đội năm 2019. Tạp chí Y học Việt Nam, 497(1), 145-149.

8. Hawamdeh M.Z, Al-Ajlouni M.J (2013). The clinical pattern of knee osteoarthritis in Jordan A hospital based study. International Journal of medical sciences, 10(6), 790-795.

\title{
TỶ LÊ HAO PHÍ VẮC XIN VÀ TIÊM CHỦNG MỞ RộNG TẠI CÁC TỈNH/THÀNH KHU VỰC PHÍA NAM VÀ CÁC YẾU TỐ LIÊN QUAN NĂM 2021
}

\author{
Phan Thị Quỳnh Trâm*, Hồ Vĩnh Thắng*, Hoàng Anh Thắng*, \\ Trịnh Trung Trực* , Võ Ngọc Quang*, Nguyễn Thị Cẩm Nhung*, \\ Lê Thị Thắng*, Đặng Ngọc Diệu*, Trần Thị Quyên*, \\ Hồ Hoàng V ũ **, Trần Thiện Thuần**.

\section{PROGRAMME OF IMMUNIZATION IN THE \\ FACTORS IN 2021} \\ SOUTHERN PROVINCE/CITIES AND RELATED
}

\section{TÓM TẮT}

Muc tiêu: Xác định tỷ lệ hao phí vắc xin trong tiêm chủng mở rộng và các yếu tố liên quan tại các tỉnh/thành khu vực Phía Nam năm 2021. Phương pháp: Thiết kễ nghiên cứu cắt ngang mô tả được thực hiện trên 90 trạm y tế xã phường tại ba tỉnh Lâm Đồng, Bình Dướng và Vĩnh Long thông qua phương pháp chọn mẫu phân tâng hệ thống từ tuyến tỉnh, huyện, xã. Kết quả và kết luận: Tỷ lệ hao phí vắc xin Sởi là 31,8\%; MR 34,4\%; DPT-VGB-Hib 0,7\%; OPV 24,6\%, DPT 56,3\%; VAT 62,9\%. Các vắc xin đống gói càng nhiều liều tỷ lệ hao phí càng cao, có mối tương quan với dân số, số đối tượng, số buổi tiêm chủng, đia hình. Vắc xin đơn liều DPT-VGB-Hib ít phụ thuộc vào những yếu tố trên. Nguyên nhân hủy lọ nguyên lọ chiếm chủ yếu là quá hạn $61 \%$, tiếp theo võ lọ $17,5 \%$. Những vắc xin đa liều có tỷ lệ hao phí cao hơn hẳn vắc xin đơn liều, vắc xin càng đống gói nhiều liêu hao phí càng cao. Tý lệ hao phí vắc xin cao ở những vùng có địa lý núi cao và̀ trung du, dân số ít, đối tượng tiêm chủng ít, những yếu tố trên này có mối tươning quan với nhau. Trong khi đó vắc xin đơn liều lại không phụ thuộc vào những yếu tố trên phụ thuộc vào quy trình bảo quản và vận chuyển và kỹ năng quản lý của cán bộ kho của cán bộ kho và nhà quản lý tiêm chủng tuyến trên. Quá hạn là nguyên nhân chủ yếu hủy nguyên lọ.

Tư khóa: Tỷ lệ hao phí vắc xin; quản lý vắc xin; tiêm chủng mở rộng; khu vực phía Nam.

\section{SUMMARY \\ WASTE RATE OF VACCINES AND EXPANDED}

*Viện Pasteur TP.HCM

**Đ̈ai hoc Y Dướ TP.HCM.

Chịu trách nhiệm chính: Phan Thị Quỳnh Trâm

Email: quynhtrampas@gmail.com

Ngày nhận bài: 29/10/2021

Ngày phản biên khoa học: 18/11/2021

Ngày duyệt bài: 19/12/2021
Objectives: Determining the vaccine wastage rate in Expanded Programme of Immunization and related factors in the southern provinces/cities in 2021. Method: Descriptive cross-sectional study design was carried out on 90 commune health stations in three provinces of Lam Dong, Binh Duong and Vinh Long through systematic stratified sampling method from provincial, district and commune levels. Result and conclusion: Measles vaccine wastage rate is $31.8 \%$; MR 34.4\%; DPT-VGB-Hib 0.7\%; OPV 24.6\%, DPT $56.3 \%$; VAT $62.9 \%$. The more the vaccines are packed with doses, the higher the wastage rate, which is correlated with the population, the number of subjects, the number of vaccination sessions, the topography, knowledge of training, and the practice of vaccine storage. The single dose DPT-VGB-Hib vaccine is less dependent on these factors. Main reason for canceling whole bottles was overdue $61 \%$, followed by broken bottles $17.5 \%$. Multi-dose vaccines have a higher waste rate than single-dose vaccines, the more doses the vaccine is packed, the higher the waste. The vaccine wastage rate is high in mountainous and midland geographical areas, low population, low vaccination population, these factors are correlated with each other. Meanwhile, single-dose vaccines do not depend on the above factors, depending on the storage and transportation procedures and the management skills of the warehouse staff of the warehouse staff and the up-line vaccination managers. Expiration is the major cause of bottle cancellations.

Key word: Vaccine wastage rate; vaccine administration; EPI; the southern area.

\section{I. ĐẶT VẤN ĐỀ}

Vắc xin là một công cụ rất hiệu quả trong dự phòng một số bệnh truyền nhiễm. Theo Tổ Chức Y Tế Thế Giới (WHO), trên thế giới vắc xin hao 
phí trên $50 \%{ }^{(1)}$. Có nhiêu yếu tố liên quan tới tỷ lệ hao phí vắc xin: dạng đóng gói của vắc xin, loại vắc xin, số đối tượng trong buổi tiêm chủng, điều kiện địa lý, mật độ dân số, hình thức tổ chức tiểm chủng, số buổi tiêm chủng/tháng, số điểm tiêm chủng/xã, phường, khả năng vận động và tập hợp đối tượng trong buổi tiêm chủng...(1, 2).

Theo báo cáo hệ số sử dụng vắc xin Khu vực Phía Nam năm 2019 - Viện Pasteur TP. Hồ Chí Minh, hệ số sử dụng vắc xin toàn khu vực đối với vắc xin 20 liều năm 2015 là từ 1,65 đến năm 2019 là 1,9 (tỷ lệ hao phí 37\% - 49\%). Trong đó, năm 2019 tỷ lệ hao phí vắc xin DPT tại Thành Phố Hồ Chí Minh là 2.7 (63\%), Kiêng Giang, Long An, Sóc Trăng, Bến Tre là 2,2 $(54 \%)^{(3,4)}$, vượt định mức cho phép theo mức quy định về hệ số sử dụng vắc xin cho vùng đồng bằng tại Quyết định 11/QĐ-VSDTTU là 1.8 $(45 \%)^{(4,5)}$. Vì muốn tìm hiểu sâu hơn những lý do trên tại địa phương chúng tôi tiến hành đề tài nghiên cứu: "Tỷ lệ hao phí vắc xin trong tiêm chủng mở rộng tại các tỉnh/thành khu vực Phía Nam và các yếu tố liên quan năm 2021" với mục tiêu: Xác định tỷ lệ hao phí vắc xin, mối liên quan về đối tượng tiêm chủng, nguyên nhân hao phí vắc xin trong tiêm chủng mở rộng tại khu vực Phía Nam năm 2021.

\section{II. ĐỐI TƯỢNG VÀ PHƯƠNG PHÁP NGHIÊN CỨU}

2.1. Thiết kế nghiên cứu: Nghiên cứu cắt ngang có phân tích định lượng.

2.2. Đối tượng nghiển cứu: Dân số mục tiêu 2639 xã/phường của 20 tỉnh/thành Khu vực Phía Nam Việt Nam. Dân số chọn mẫu là 347

\section{KẾT QUẢ NGHIÊN CỨU}

Bảng 1. Tỷ lệ hao phí vắc xin của các vắc xin tại tỉnh Bình Dương, Vĩnh Long, Lâm Đồng năm $2021(N=90)$

\begin{tabular}{|c|c|c|c|c|c|}
\hline Loại vắc xin & $\begin{array}{c}\text { Số } \\
\text { liều/lọ }\end{array}$ & Đường dùng & $\begin{array}{c}\text { Số liều } \\
\text { được mở }\end{array}$ & $\begin{array}{c}\text { Số đối tượng } \\
\text { được tiêmm }\end{array}$ & $\begin{array}{c}\text { Tỷ lệ hao } \\
\text { phí }\end{array}$ \\
\hline Sởi* & 10 & Tiêm & 13.380 & 10.027 & $31,8 \%$ \\
\hline MR* & 10 & Tiêm & 9.570 & 6.808 & $34,4 \%$ \\
\hline DPT-VGB-Hib\# & 1 & Tiêm & 32.220 & 32.192 & $0,7 \%$ \\
\hline OPV\# & 20 & Uống & 37.070 & 29.959 & $24,6 \%$ \\
\hline DPT\# & 20 & Tiêm & 4.640 & 3.022 & $56,3 \%$ \\
\hline VAT\# & 20 & Tiêm & 9.870 & 5.088 & $62,9 \%$ \\
\hline
\end{tabular}

\# Dạng dung dịch; *Dạng đông khô

Tỷ lệ hao phí vắc xin dạng nước đa liêu (20 liêu/lọ) có tỷ lệ hao phí cao nhất VAT_62,9\% và DPT_56,3\% , tiếp đó là vắc xin dạng đông khô đa liều (10 liều/lọ) MR_34,4\%; Sởi_31,8\%. Tỷ lệ hao phí thấp nhất là vắc xin dạng dung dịch một liêu chiếm DPT-VGB-Hib_0,7\%. Tỷ lệ hao phí càng cao trạm y tế xã/phường của tỉnh Bình Dương (trung du), Vĩnh Long (đồng bằng), Lâm Đồng (miên núi).

2.3. Cỡ mâuu: Chọn cỡ mẫu xã/phường: sau khi phân tần và chọn ngẫu nhiên đơn chúng tôi chọn 30 xã/phường cho mỗi tỉnh/thành. Tính được cõ̃ mẫu là 90 xã/phường.

Tiêu chuẩn lựa chọn: Trạm Y tế xã/phường có thực hiện tiêm chủng mở rộng thường xuyên hàng tháng.

Tiêu chuẩn loại bỏ: Các Trạm Y Tế đang thực hiện tiêm chiến dịch vắc xin; những trường hợp khổng đồng ý tham gia.

\subsection{Thời gian và địa điểm nghiên cứu:}

Thời gian nghiên cứu: Tháng 1 năm 2021tháng 9 năm 2021

Địa điểm nghiên cứu: 90 xã/phường tại 15 huyện thuộc 3 tỉnh Vính Long, Bình Dương và Lâm Đồng.

2.5. Thu thập dữ kiện: Phương pháp thu thập số liệu: Thu thập thổng tin được lấy từ hồ sơ lưu trữ dưới dạng các báo cáo vắc xin và vật tư tiêm chủng hàng tháng và các sổ quản lý vắc xin và vật tư tiêm chủng hàng tháng, sổ tiêm chủng mở rộng hàng tháng, báo cáo năm , kế hoạch tiêm chủng mối hàng tháng, quý, năm tại 90 trạm y tế tuyến xã/phường. Công cụ thu thập số liệu: Dùng bảng câu hỏi soạn sẵn để thu thập số liệu và phỏng vấn cán bộ y tế.

2.6. Xử lý số liệu: số liệu được làm sạch và nhập bằng chương trình Epidata Manager và phần mềm Excel Microsoft Excel 2013, phân tích bằng chương trình STATA 14.0.

2.7. Vấn đề $\mathbf{Y}$ Đức: Nghiên cứu được thực hiện thông qua sự chấp thuận bởi hội đồng đạo đức của Đại học Y Dược thành phố Hồ Chí Minh. hơn những vắc xin đống gói càng nhiều liều.

Tỷ lệ hao phí vắc xin theo đối tượng tiêm chủng và mối liên quan. Kết quả cho thấy đối tượng càng giảm thì tỷ lệ hao phí vắc xin càng cao, điêu này khá rõ ở tất cả các vắc xin đa liều dạng dung dịch, dạng đông khô theo 
phân loại dạng trình bày. Có sự tương quan ( $\mathrm{r}=-$ $0,5 ; p=0,000)$ giữa yếu tố số đối tượng uống vắc xin $O P V$ và tỷ lệ hao phí vắc xin $O P V$. Số đối tượng uống vắc xin OPV có thể giải thích $14 \%$ $\left(R^{2}=0,14\right)$ sự thay đổi về tỷ lệ hao phí vắc xin OPV, phương trình hồi quy theo phân vị (Quantile regression) cho thấy số đối tượng uống OPV tăng lên 1 đơn vị (300-500 đối tượng) thì tỷ lệ hao phí sẽ giảm đi 7,5\%.

Có sự tương quan $(r=-0,4 ; p=0,032)$ giữa yếu tố số đối tượng tiêm và tỳ lệ hao phí vắc xin DPT. Số đối tượng tiêm vắc xin DPT có thể giải thích $10 \%(R 2=0,1)$ sự thay đổi về tỷ lệ hao phí, phương trình hồi quy theo phân vị (Quantile regression) cho thấy số đối tượng tiêm DPT tăng lên 1 đơn vị (200 đối tượng) thì tỷ lệ hao phí sẽ giảm đi $15 \%$.

Có sự tương $(r=-0,5 ; p=0,000)$ giữa yếu tố số đối tượng tiêm vắc xin Sởi và tỷ lệ hao phí. Số đối tượng tiêm vắc xin Sởi có thể giải thích $11,4 \%(R 2=0,114)$ sự thay đổi về tỷ lệ hao phí vắc xin Sởi, phương trình hồi quy theo phân vị (Quantile regression) cho thây số đối tượng tiêm Sởi tăng lên 1 đơn vị (200 đối tượng) thì tỷ lệ hao phí sẽ giảm đi 9,4\%.

Có sự tương quang $(r=-0,43 ; p=0,000)$ đối với yếu tố tổng đối tượng tiêm vắc xin $M R$ và tỷ lệ hao phí). Số đối tượng có thể giải thích 17,4\% (R-squared $=0.174$ ) sự thai đổi của tỷ lệ hao phí vắc xin $M R$, phương trình hồi quy cho thấy số đối tượng tăng lên 1 đơn chị (200 đối tượng) thì tỷ lệ hao phí sẽ giảm đi 9,7\%.

Có sự tương quan $(r=-0,7 ; p=0,000)$ giữa yếu tố số đối tượng tiêm vắc xin VAT và tỷ lệ hao phí. Số đối tượng tiêm vắc xin VAT có thể giải thích $32,7 \%(R 2=0,327)$ sự thay đổi về tỷ lệ hao phí vắc xin VAT, phương trình hồi quy theo phân vị (Quantile regression) cho thây số đỗi tượng tiêm VAT tăng lên 1 đơn vị (200 đối tượng) thì tỷ lệ hao phí sẽ giảm đi 30,1\%.

\section{BÀN LUÂ̂N}

Đối với vắc xin đơn liều DPT-VGB-Hib, tỷ lệ hao phí vắc xin chung thực tế thấp hơn định mức cho phép $(0,7 \%)$ so với Quyết định 1193/QĐVSDTTƯ ký ngày $10 / 9 / 2019$ là $5 \%(1.05)^{(6)}$, và thấp hơn định mức cho phép của WHO là $5 \%{ }^{(1)}$, thấp hơn tỷ lệ hao phí hầu hết các nghiên cứu như đánh giá hao phí vắc xin tại Ethiopia năm 2020 là $7 \%(7)$, và đánh giá về quản lý vắc xin tại vùng Bắc Trung Tâm Nigenia là $23 \%{ }^{(8)}$. Điều này cho thấy công tác quản lý vắc xin và bảo quản, vận chuyển dây truyên lạnh tại tuyến xã்/phường, cũng như công tác quản lý dây chuyên lạnh từ tuyến trên là khá tốt, hao phí vắc xin hầu như là không. Muốn tối đa giảm hao phí vắc xin này cần cải thiện và quản lý tốt hệ thống dây chuyền lạnh đầu tư hệ thống tủ lạnh chuyên dụng ở các tuyến, cũng như phương tiện chuyên về vận chuyển, hệ thống đọc nhiệt độ liên tục như các Fridge-tag điện tử, cải thiện quản lý kho từ tuyến trung ương đến địa phương, cũng như kỹ năng của cán bộ y tế.

Vắc xin đa liêu dạng tiêm DPT tiêm cho trẻ 18-24 tháng tuổi trong Chương trình tiêm chủng mở rộng, dạng đóng gói 20 liều/lọ. Ghi nhận hao phí $56,3 \%$, kết quả này là cao do với tiêu chuẩn giới hạn mức cho phép của Bộ Y Tế và của WHO là $55 \%^{(6)}(2.2)$ và $50 \%{ }^{(1)(2)}$. Mức hao phí này cao hơn so với các nghiên cứu tai India Delhi 16\%(1.19); Nigeria 15\%(1.18); Ethiopia 12,67\% $(1.2)^{(7)}$. Nếu xét về từng vùng địa lý, vùng đồng bằng và trung du là vượt định mức là $70 \%$, $54,2 \%$, so với định mức cho phép của Bộ Y tế $50 \%$. Chỉ có miền núi (56\%) đúng định mức $(70 \%)$ kết quả này là khá phù hợp với nghiên cứu " Thực trạng hệ số sử dụng vắc xin trong tiêm chủng mở rộng tại 20 tỉnh/thành Phía Nam, năm 2018" số liệu của nghiên cứu này chỉ rất rõ các tỉnh đồng bằng (Vính Long) và tỉnh trung du (Bình Dương) hệ số sử dụng vượt định mức $>2$ (>50\%), tỉnh miền núi (Lâm Đồng) hệ số sử dụng là đứng định mức ${ }^{[2)}$. Theo nhóm nghiên cứu nên giảm liêu vắc xin từ 20 liều/lọ thành 10 liêu/lo từ đó tối đa hóa hao phí vắc xin.

Vắc xin dạng dung dịch đa liều VAT 20 liều/lọ tiêm cho phụ nữ có thai và phụ nữ ở độ tuổi sinh đẻ. Nghiên cứu cho thây hao phí cao nhất trong 6 lại vắc xin được nghiên cứu $(62,9 \%)$, kết quả này cao hơn định mức của Bộ $Y$ tế là $45 \%$ $(2.2)^{(6)}$, và cao hơn định mức của WHO là $50 \%{ }^{(1)}$, số liệu này còn cao hơn nghiên cứu về hệ số sử dụng Khu Vực Phía Nam, Việt Nam là $45 \%(1.7)^{(2)}$. Miền núi là nơi có tỷ lệ hao phí cao nhất $(66,7 \%)$ và hao phí giảm dân qua các vùng miền trung du và đồng bằng, khoảng cách xa, dân số ít và đối tượng tiêm chủng thấp, chia nhiều buổi tiêm chủng trong tháng cũng làm tăng hao phí vắc xin. Cán bộ y tế được tập huấn tốt cũng làm giảm hao phí. Kiến nghị có thể thay thế lọ vắc xin VAT từ 20 liều/lọ thành 10 liều/lọ để tối đa hóa tỷ lệ sử dung vắc xin trong các lọ mở. Phụ thuộc rất nhiều dân số, đối tượng tiêm chủng, địa hình, kỹ năng tổ chức buổi tiêm chủng, số buổi tiêm chủng cũng như kỹ năng quản lý bảo quản cán bộ y tế tuyến xã/phường.

Việc quản lý được đối tượng mà cụ thể là nắm được số trẻ có mặt tại địa phương là việc 
rất quan trong và cũng là công việc khó khăn nhất ở những vùng dân cư có mật độ di biến động lớn, đặc biệt là những thành phố đô thị lớn và khu công nghiệp.

\section{KẾT LUÂ̂N}

Các vắc xin đống gói càng nhiều liều tỷ lệ hao phí càng cao, có mối tương quan với dân số, số đối tượng, số buổi tiêm chủng, địa hình. Vắc xin đơn liều DPT-VGB-Hib ít phụ thuộc vào những yếu tố trên. Có thể giảm liều đống gói vắc xin VAT và DPT từ 20 liêu/lọ thàng 10 liều/lọ. Cần đầu tư và duy trì hệ thống bảo quản, vận chuyển dây chuyền lạnh chuyên dụng từ tuyến trung ương đến địa phương. Cán bộ quản lý tiêm chủng cần quản lý tốt đối tượng của địa phương, nắm rõ dân số, số đối tượng, đặc tính dân số, kỹ năng tổ chức buổi tiêm chủng. Kết quả nghiên cứu là cơ sở đưa ra các khuyến nghị giúp tỉnh/thành triển khai công tác quản lý vắc xin tiêm chủng mở rộng tốt hơn và tìm giải pháp giảm hao phí vắc xin.

\section{TÀI LIẸU THAM KHẢO}

1. Organization WH. Monitoring vaccine wastage at country level: guidelines for programme managers. Geneva: World Health Organization; 2005.
2. Trịnh Trung Trực*. Võ Ngọc Quang TQT, Nguyễn Thi Thu Trang, Hoàng Anh Thắng, Phan Thị Quỳnh Trâm, Hồ Vình Thắng. Thực trạng hệ số sử dụng vắc xin trong Tiêm chủng mở rộng tại 20 tỉnh/thành KVPN năm 2018. Tạp chí Y Học Dự Phòng. 2019:48.

3. Minh VPTCMRKVPN-VPTpHC. Báo cáo hê số sử dụng vắc xin Khu vực Phía Nam từ năm 2015 đến nằm 2019. Viên Pasteur Thành Phố Hồ Chí Minh. 2019.

4. Báo cáo Tiềm chủng mở rộng Khu vức Phía Nam năm 2015. Viện Pasteưr Thành Phố Hồ Chí Minh. 2015.

5. Viện Vệ sinh dịch tễ Trung ương (2017) Quyết định số $11 / \mathrm{Q} Đ-V S D T T U$ V Vê việc phê duyệt định múcc sử dụng, dự trử vắc xin vật tư Tiêm chủng trong dự án Tiêm chủng mở rộng. Thư Viện Pháp Luât.

6. Viện Vệ sinh dịch tễ Trung ương (2019) Quyết định 1193/Q̄Đ-VSDTTU' "điều chỉnh mức sứ dụng, dự trữ vắc xin và vật tư tiêm chủng trong Dự an Tiêm chủng mở rộng". Bộ Y Tế. 2019.

7. Mohammed SA, Workneh BD. Practical experience of vaccinators and vaccine handlers in vaccine cold chain management: A phenomenological study. Ethiopian Journal of Health Development. 2021;35(1).

8. Kure BC, Olugbenga OT, Mamzhil R, Crown S, Paul BA, Sabuwa BH, et al. Vaccine Management in North Central Nigeria: A Review of the Impact of Optimized Integrated Routine Immunization System, Kaduna State, Nigeria. 2019.

\title{
TỶ LÊ TIÊM CHỦNG ĐÂYY ĐỦ, ĐÚNG LICCH CỦA TRẺ EM THEO CHƯO'NG TRÌNH TIÊM CHỦNG MỞ RộNG VÀ CÁC YẾU TỐ LIÊN QUAN TẠI THÀNH PHỐ PHAN THIẾT, TỈNH BÌNH THUÂ̂N
}

\author{
Vũ Thị Thúy*,Võ Văn Hạn*, Nguyễn Thị Tho*,
} Hồ Hoàng Vũ $\tilde{u}^{* *}$,Trần Thiện Thuần**

\section{TÓM TẮT}

Mục tiêu: Xác định tỷ lệ trẻ dưới 1 tuổi được tiêm chủng đầy đủ, đúng lịch 8 loại vắc xin và các yếu tố liên quan tại thành phố Phan Thiết, tỉnh Bình Thuận trong năm 2020. Phương pháp: Thiết kế nghiên cứu cắt ngang mô tả trên 370 trẻ emtrong độ tuổi $9-21$ tháng tuổi đến $31 / 12 / 2020$ đang sống tại thành phố Phan Thiết, tỉnh Bình Thuận đến thời điểm nghiên cứu và bà me (người trực tiếp chăm sóc trẻ) trên 18 tuổi đang sống taii thành phố Phan Thiết, tỉnh Bình Thuận đến thời điểm nghiên cứu thông qua phương pháp chọn mâu PPS, chọn ngâu nhiên 30 cụm (đơn vị cụm

\footnotetext{
*Trung tâm Kiểm soát bệnh tật Bình Thuận

**Đai hoc Y Dước TP Hồ Chí Minh

Chịu trách nhiệm chính: Vũ Thị Thúy

Email: thuyvut4g@gmail.com

Ngày nhận bài: 29/10/2021

Ngày phản biên khoa họ: 25/11/2021

Ngày duyệt bài: 17/12/2021
}

là 121 thôn/khu phố của 17 xã/phường của thành phố Phan Thiết). Kết quả: Tỷ lệ tiêm chủng đầy đủ, tại thành phố Phan Thiết, tỉnh Bình Thuân trong năm 2020 là $78.11 \%$, tiêm chủng đúng lịch là $1.98 \%$. Các yếu tố liên quan đế tỷ lệ tiêm chủng đầy đủ 8 loại vắc xin cho trẻ dưới 1 tuổi được nhận định bao gồm: Trình độ học vấn; hết cấp 2 PR:1.23, KTC 95\%(1.01-1.50) $(p=0.01)$ hết cấp3PR: 1.27 KTC $95 \%(1.05-1.54)$ $\mathrm{p}=0.04$, trên cấp 3 PR: 1.23 KTC 95\%(1.01-1.49) $p=0.01$. Nơi ở, vung ven thành thị PR: $1.23 \mathrm{KTC} 95 \%$ (1.11-1.36) $p=0.00$. Sổ tiêm chủng PR 4.81 KTC $95 \%$ (1.36-17.06) $\mathrm{p}=0.00$; Biết nđ̛i, tiêm giờ tiêm PR 2.38 KTC 95\% (1.07-5.32) $\mathrm{p}=0.00$; trẻ bi hoãn tiêm PR 0.84 KTC $95 \%(0.72-0.98) p=0.01$, Không tin vào tiêm chủng PR 0.83 KTC 95\% (0.7-0.98) $p=0.01$; nghe đồn không đúng về tiêm chủng PR 0.76 KTC $95 \%$ (0.60$0.97) p=0.00$; kết vắc xin $P R=0.81 K T C 95 \%(0.67-$ 0.97) $\mathrm{p}=0.01$; mẹ bận PR 0.77 KTC $95 \%$ (0.63-0.95) $p=0.00$, trẻ bệnh PR 0.85 KTC $(0.77-0.95) p=0.00$, lựa chọn nơi tiêm PR 2.23 KTC 95\% $(1.86-2.66) \mathrm{p}=0.00$. Kểt luận: Tỷ lệ tiêm chủng đầy đủ, 8 loại vắc xin truong chương trình tiêm chủng mở rộng là $78.11 \%$, 